\title{
Supporting Information: Pore formation in silicon nanoparticle thin films and its impact on optical properties
}

N. Vulic, ${ }^{\dagger}$ J.V. Carpenter III, ${ }^{\ddagger}$ P. Firth, ${ }^{\dagger}$ N. Rodkey,${ }^{\dagger}$ Z.C. Holman, ${ }^{\dagger}$ and S.M. Goodnick ${ }^{*, \dagger}$

$\dagger$ School of Electrical Computer and Energy Engineering, Arizona State University, Tempe, Arizona, 85281, USA

$\ddagger$ School for the Engineering of Matter, Transport, and Energy, Arizona State University, Tempe, Arizona, 85281, USA

E-mail: stephen.goodnick@asu.edu

\section{Ellipsometry fits for different porosities}

The ellipsometry fits to the EMA model are shown in Fig. S1 for the $\sim 70 \%, \sim 80 \%$, and $\sim 90 \%$ porous SiNP thin films. For the fits shown in (a)-(c), the model does not take into account surface roughness. The corresponding uniqueness of fits graphs in (d)-(f), however, include surface roughness. Nevertheless, for the $\sim 70 \%$ and $\sim 80 \%$ samples, minimized RMSE value agrees well with the optimized model that did not take into account surface roughness (shown by the vertical dashed line). For the $\sim 90 \%$ sample, the effect of surface roughness is more pronounced. An improved fit is achieved by considering the surface roughness parameter, as shown in Fig. S2. 
(a)

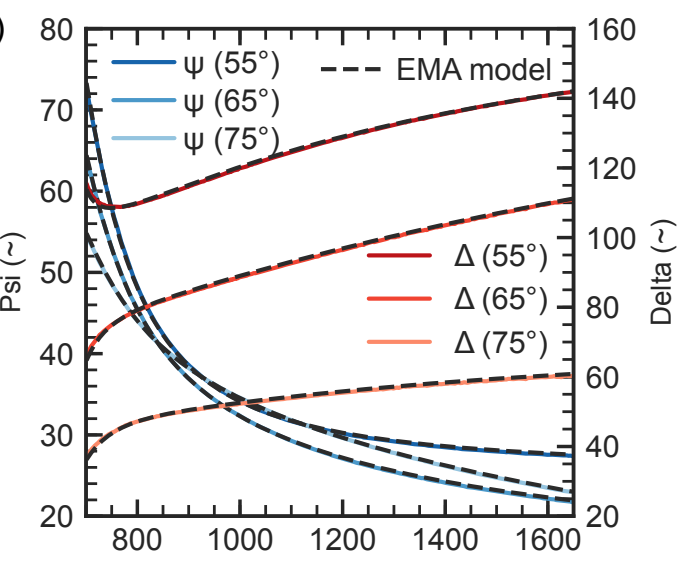

(b) 50 160

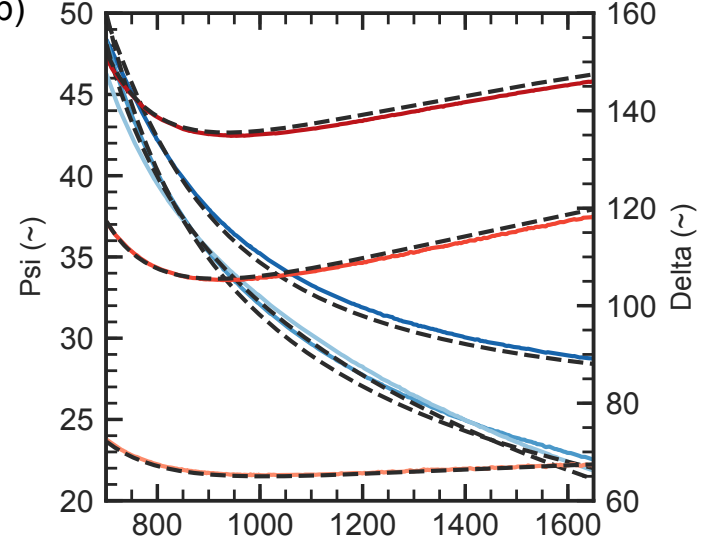

(c) 50 , 210

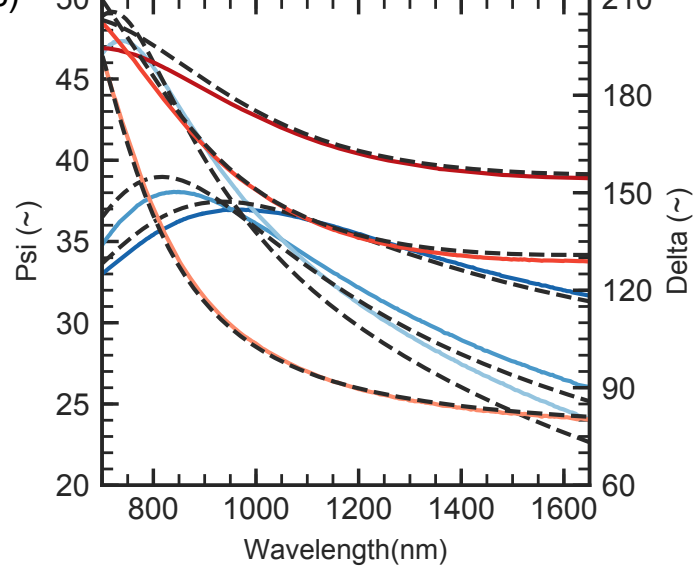

(d)

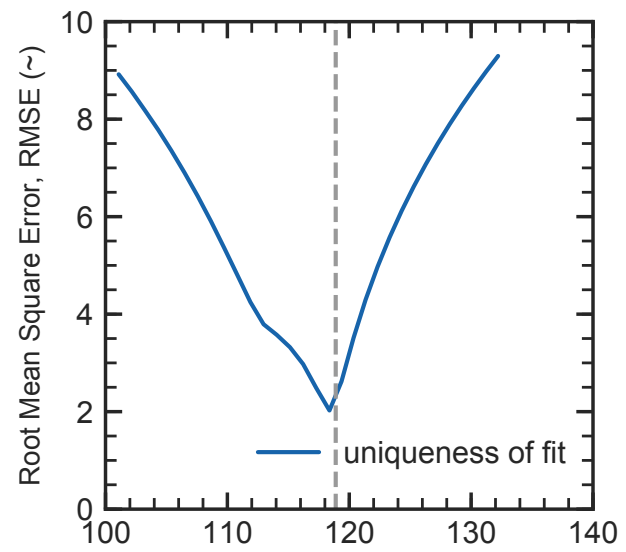

(e)

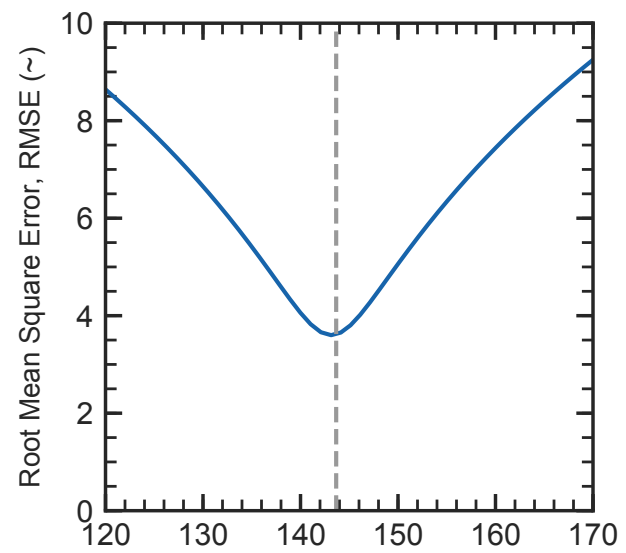

(f)

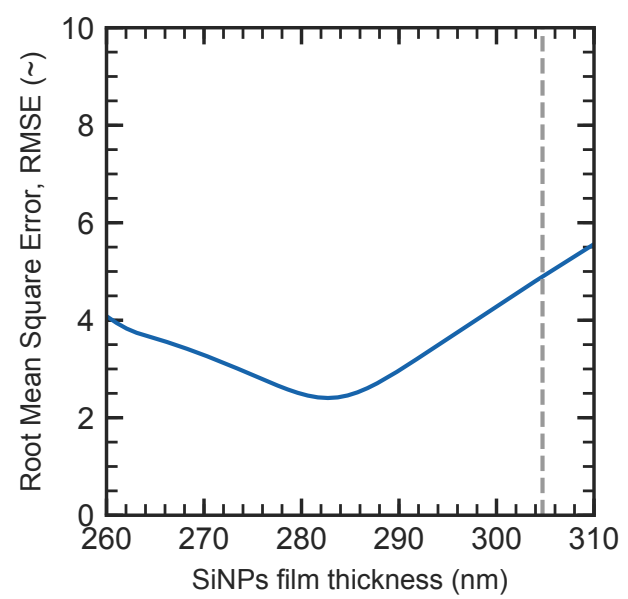

Figure S1: (a)-(c) Ellipsometry fits of $\sim 70 \%, \sim 80 \%$, and $\sim 90 \%$ porous SiNP thin films at three different VASE angles. (d)-(f) Corresponding uniqueness of fit graphs, with the dashed line indicating the location for the fits shown. 
(a)

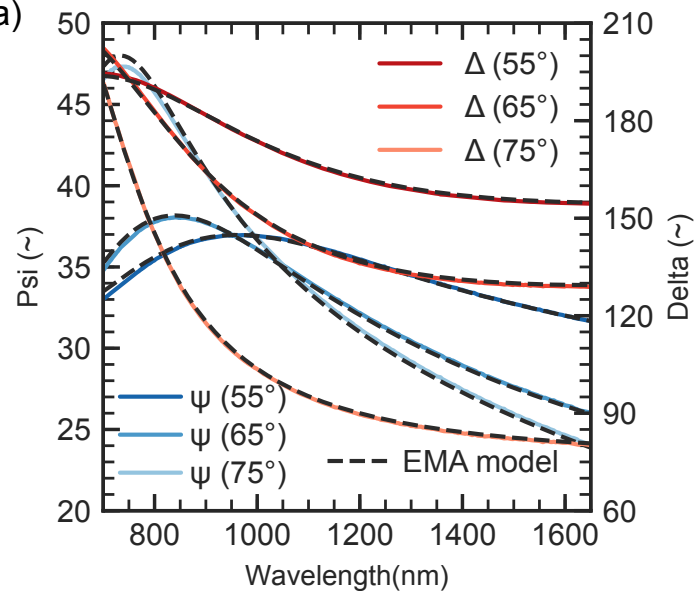

(b)

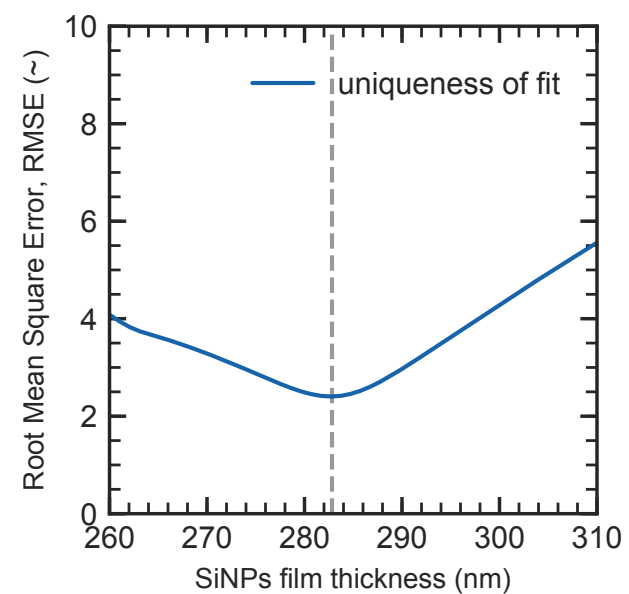

Figure S2: (a) Ellipsometry fit for $\sim 90 \%$ porous SiNP thin films with included surface roughness at three different VASE angles, and (b) the corresponding uniqueness of fit graph, with the dashed line indicating the location for the fit shown (thickness of $282.71 \mathrm{~nm}$, surface roughness of $160.32 \mathrm{~nm}$, and a porosity of $89.3 \%$ ).

\section{Pore size distribution from cross-sectional SEM images}

The SEM images are not the perfect slices of the nanoparticle layer, and, as a result, underestimate the volumetric porosity as more particle area is shown from the rounded surfaces. The 2D porosities of the thresholded SEM images in Fig. S3(a) are 13.55\%, 38.14\%, and $68.35 \%$, respectively. Similarly, from Fig. S3(c), the 2D porosities are $27.41 \%, 50.58 \%$, and $75.06 \%$, respectively. While the $2 \mathrm{D}$ porosities underestimate the volume porosity, they follow the same trend as volumetric porosities obtained from the EMA model and the BET nitrogen adsorption measurements. This trend is more readily observed when comparing their 2D densities (100\%-Porosity). In addition, the 2D densities are around 2.5 to 3 times higher than the corresponding volumetric densities obtained using other methods, further suggesting that we are observing multiple layers. 
(a)

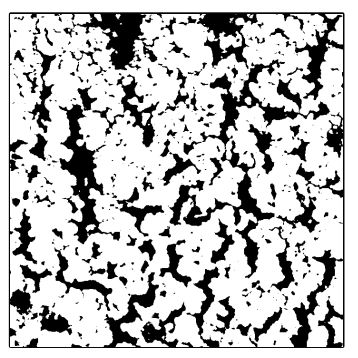

(b)
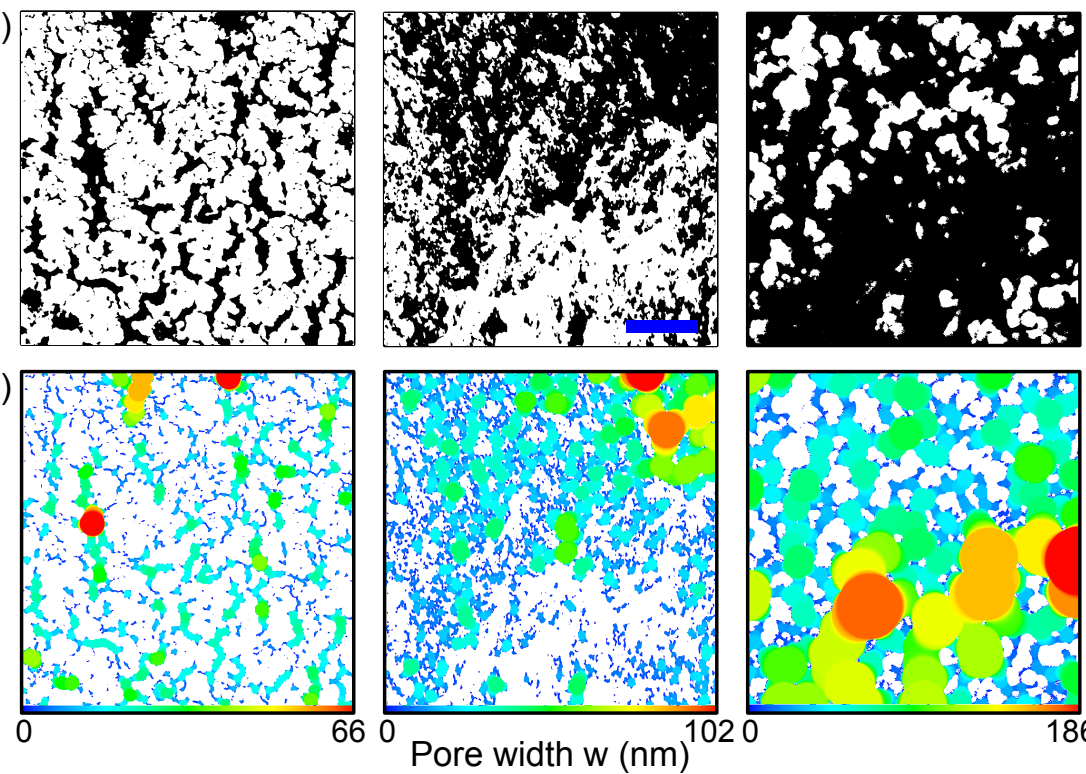

(c)

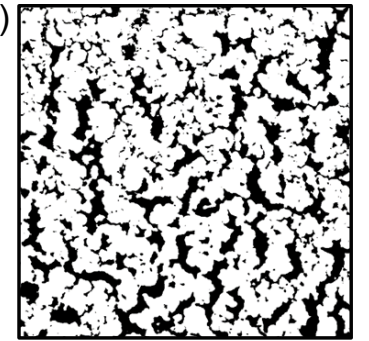

(d)
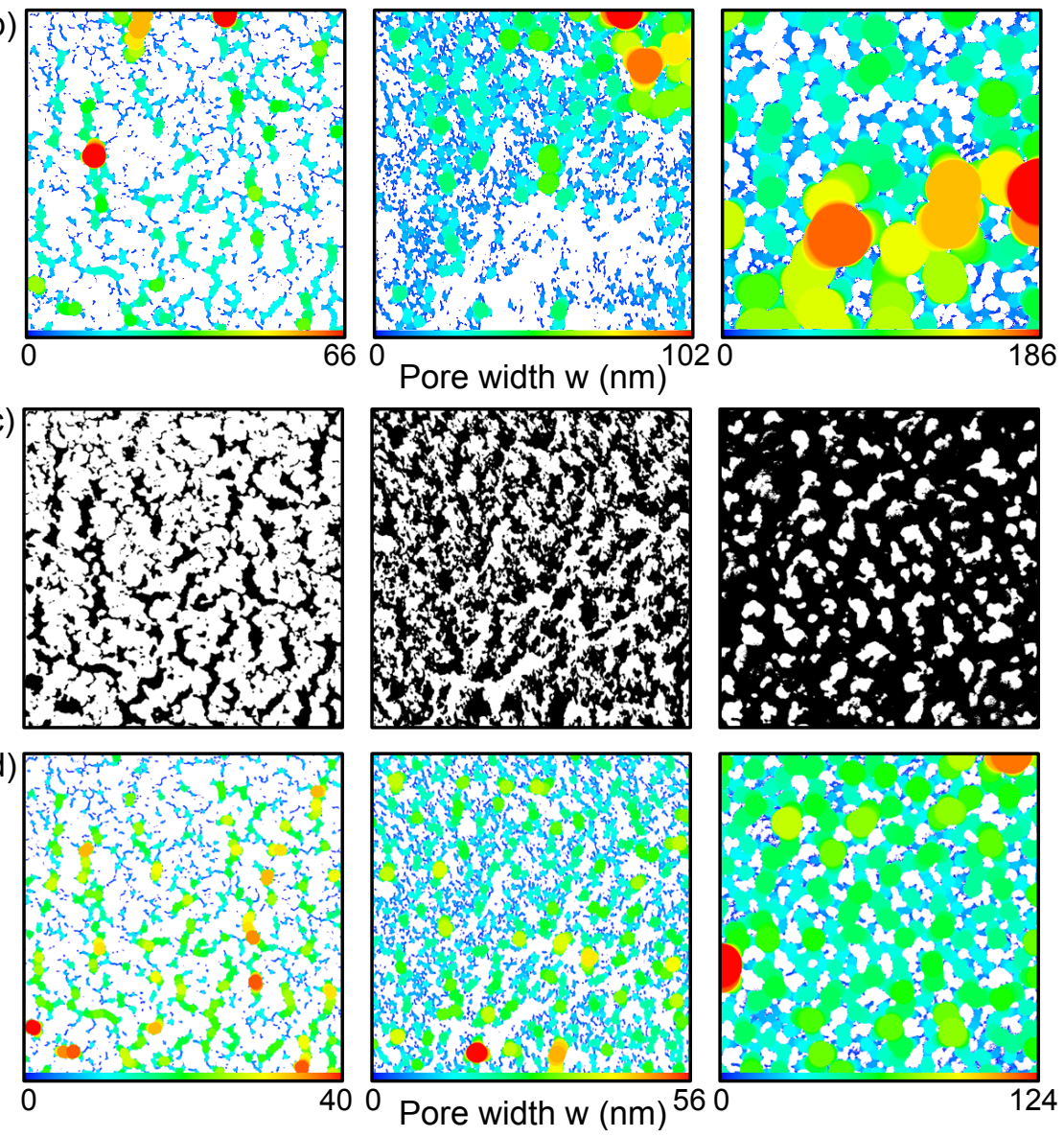

Figure S3: SEM cross-section images using (a) global and (c) local thresholding and the corresponding pore size distributions in (b) and (d). Scale bar corresponds to $200 \mathrm{~nm}$. 


\section{BET pore size distribution, tail-end}

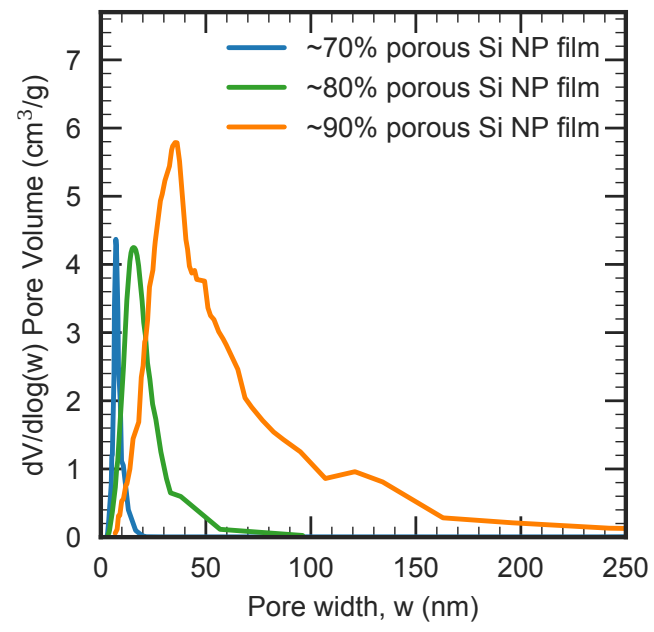

Figure S4: BET pore size distribution showing the tail extension of the $\sim 90 \%$ porous film.

\section{RTA of the thickest Si nanoparticle films}

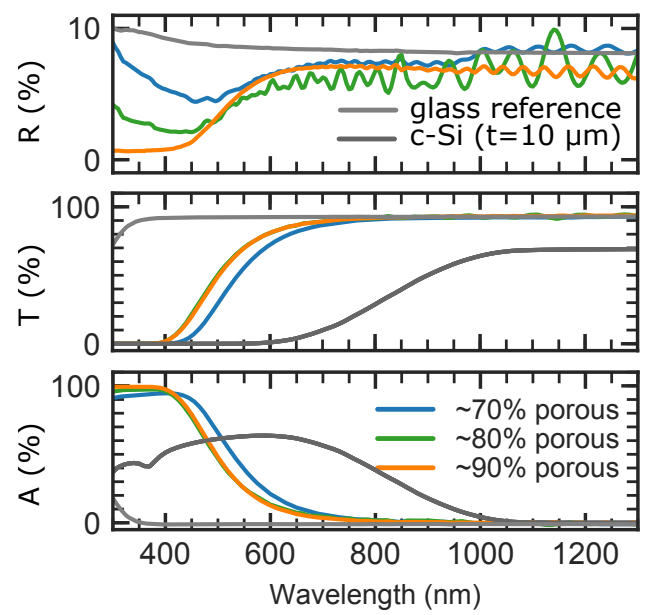

Figure S5: Reflectance (R), transmittance ( $\mathrm{T}$ ) and absorbtance (A) of thick silicon nanoparticle films $\left(\mathrm{t}_{\mathrm{eff}} \approx 1.5 \mu \mathrm{m}\right)$ for which haze, angular intensity distribution, and volumetric pore size distribution are measured. 


\section{Angle-resolved measurements}

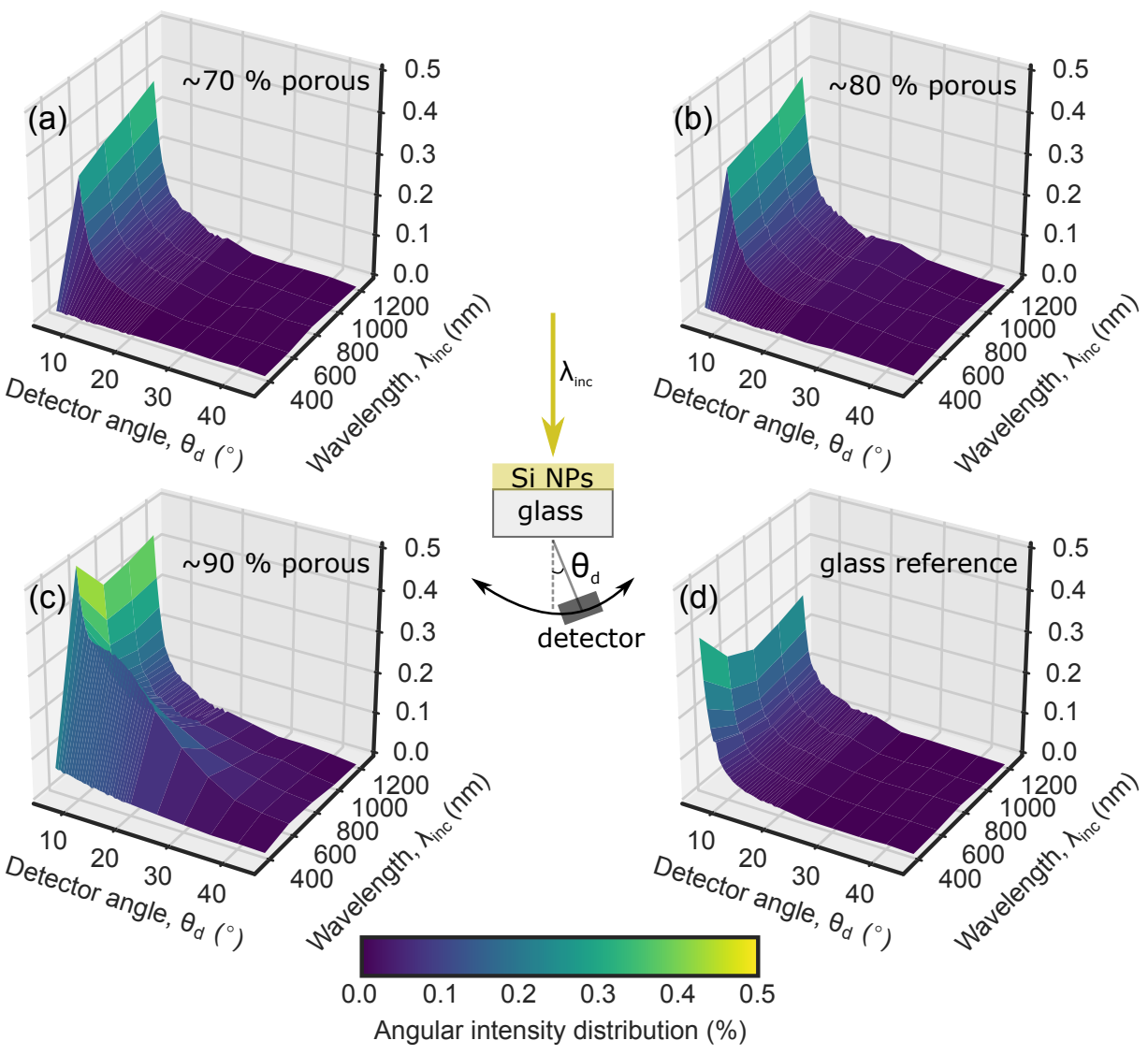

Figure S6: Angle-resolved intensity scattering in transmission through Si nanoparticles films of increasing porosity from (a) to (c) deposited on glass substrates [glass reference is shown in (d)]. The incoming light is normal to the samples and is varied in energy. We observe an increase in non-specular transmission (broader scattering angles) with increasing porosity. These trends agree with the measured haze values.

While transmission haze compares the diffuse scattering integrated along all angles within a hemisphere (except the aperture window for direct transmission) and compares it to the total transmission through the sample (Fig. 5 of the main text), angular intensity distribution (AID) measurements provide transmission intensities at different angles within a single horizontal plane. As a result, the values we obtain using AID are significantly lower than those we obtain with haze. Figures S6(a)-(c) show the corresponding AID of the nanoparticle samples, with the glass substrate shown for reference in d. With AID measurements, 
we are still able to observe similar trends with increase in porosity as we did with haze measurement, although small differences are harder to detect. Both $70 \%$ and $80 \%$ porous samples exhibit low levels of scattering, but no differences are apparent between their two angular distributions. The $90 \%$ porous film shows increased scattering across the full wavelength range, especially in the 400-800 $\mathrm{nm}$ region. These results are consistent with our observations in Fig. 5 of the main text. 


\section{Reference glass substrate: measured vs. simulated}

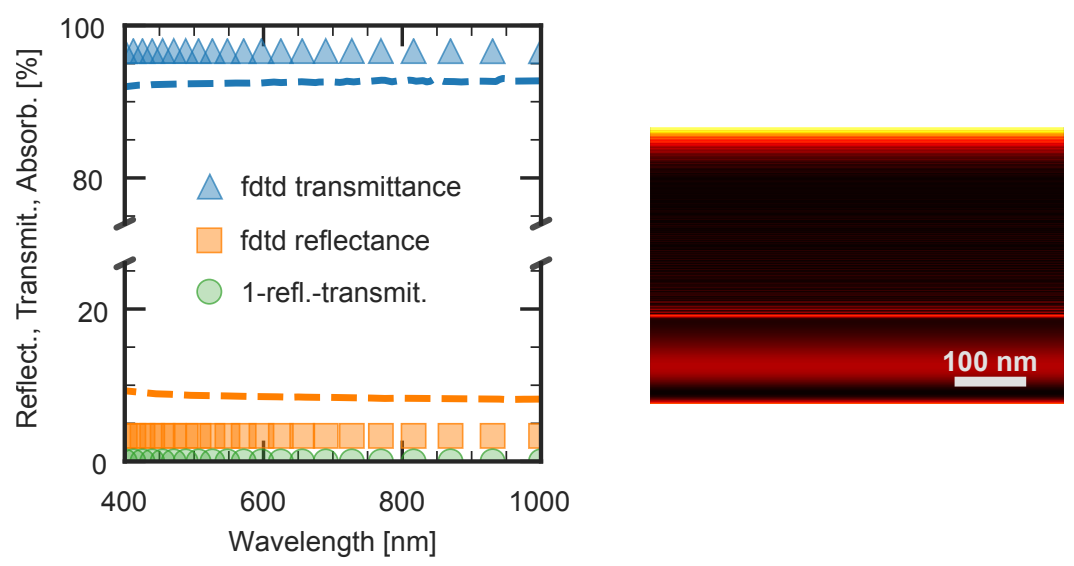

Figure S7: Measured (dashed lines) vs. simulated (symbols) RTA for the glass reference. The electric field energy density is included on the right.

\section{Angle-resolved simulation of clustered structures}

Figure S8 shows the simulated angular intensity distribution of the corresponding clustered structures with the air reference shown in the first frame. The full detection-angle range, shown in (a), highlights the differences in direct transmission between the structures, similar to what is observed in the near-field. In (b), for the reduced detection-angle range $\left(\theta_{d}>7.5\right.$ degrees), similar features are observed for the three samples, with no clear indication of differences in scattering. The ripples observed in these graphs arise from the dimension of the near-field monitor (600 nm x $600 \mathrm{~nm}$ ), which, physically, acts like an aperture causing strong diffraction. The final result plotted here is the phase-corrected sum of 100 periods, which significantly reduces the broadening arising from diffraction. 

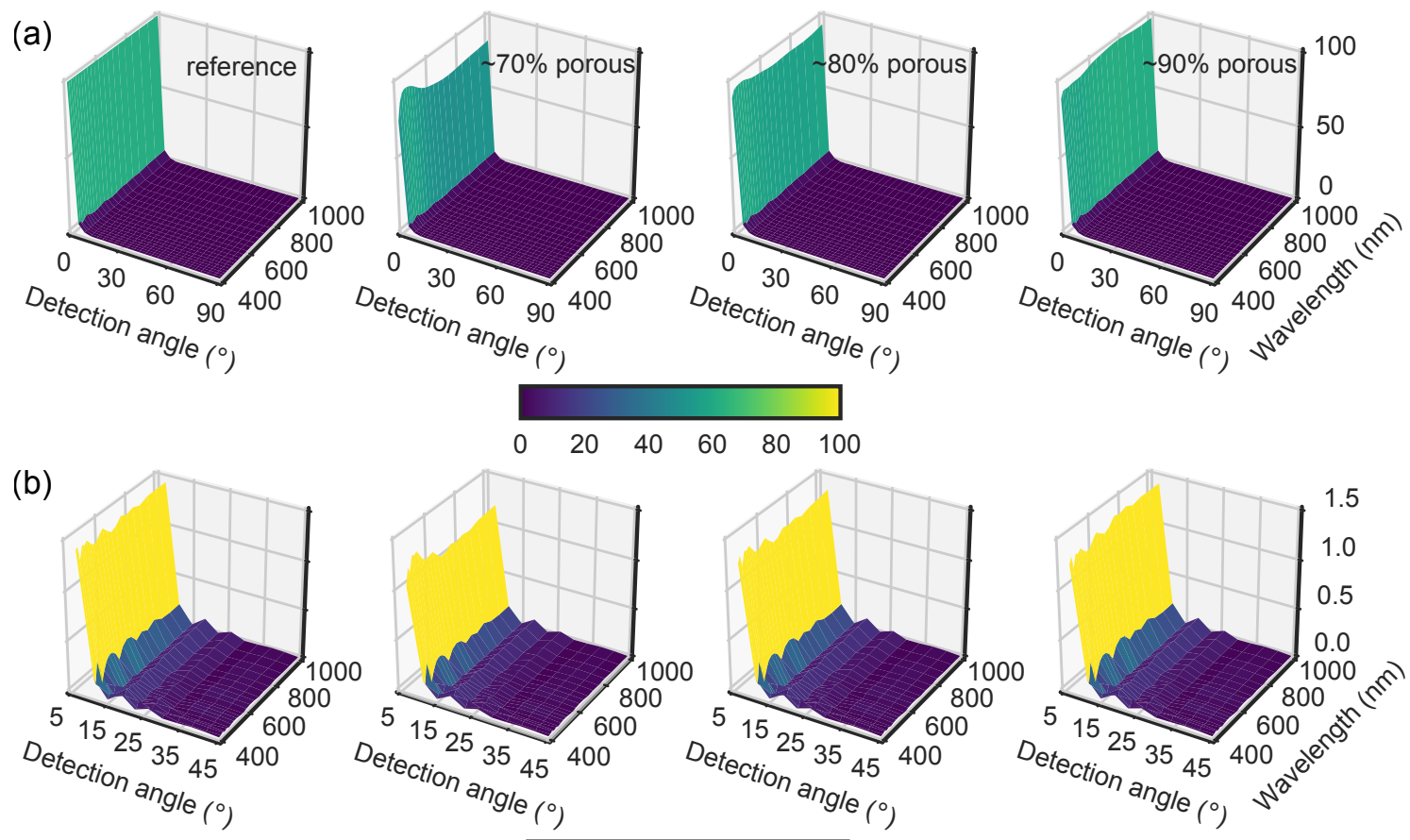

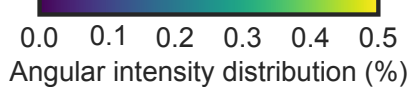

Figure S8: Simulated angular intensity distribution in transmission of the $\sim 70 \%, \sim 80 \%$, and $\sim 90 \%$ clustered nanoparticle structures for the simulated film thicknesses of 119, 144, and $305 \mathrm{~nm}$, respectively in (a) the full range of detection angles with respect to the normal and (b) detection angles above 7.5 degrees. 